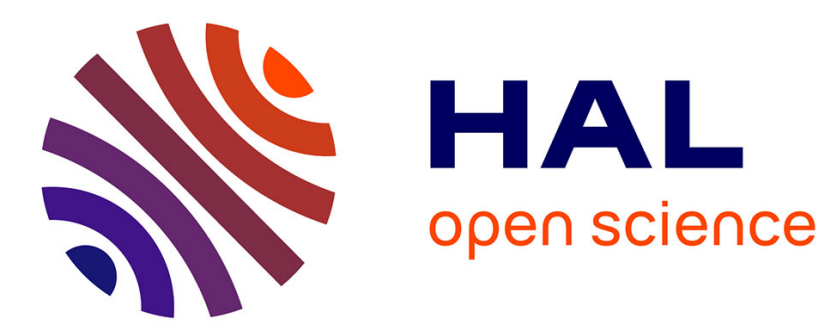

\title{
Propriété industrielle et santé publique
}

Maurice Cassier

\section{To cite this version:}

Maurice Cassier. Propriété industrielle et santé publique. Revue Projet, 2002, 270 (2), 10.3917/pro.270.0047. halshs-01893992

\section{HAL Id: halshs-01893992 \\ https://shs.hal.science/halshs-01893992}

Submitted on 11 Oct 2018

HAL is a multi-disciplinary open access archive for the deposit and dissemination of scientific research documents, whether they are published or not. The documents may come from teaching and research institutions in France or abroad, or from public or private research centers.
L'archive ouverte pluridisciplinaire HAL, est destinée au dépôt et à la diffusion de documents scientifiques de niveau recherche, publiés ou non, émanant des établissements d'enseignement et de recherche français ou étrangers, des laboratoires publics ou privés. 


\title{
PROPRIÉTÉ INDUSTRIELLE ET SANTÉ PUBLIQUE
}

\author{
Maurice Cassier
}

C.E.R.A.S | Projet

$2002 / 2-n^{\circ} 270$

pages 47 à 55

\section{ISSN 0033-0884}

Article disponible en ligne à l'adresse:

http://www.cairn.info/revue-projet-2002-2-page-47.htm

Pour citer cet article :

Cassier Maurice, « Propriété industrielle et santé publique »,

Projet, 2002/2 n²70, p. 47-55. DOI : 10.3917/pro.270.0047

Distribution électronique Cairn.info pour C.E.R.A.S.

(c) C.E.R.A.S. Tous droits réservés pour tous pays.

La reproduction ou représentation de cet article, notamment par photocopie, n'est autorisée que dans les limites des conditions générales d'utilisation du site ou, le cas échéant, des conditions générales de la licence souscrite par votre établissement. Toute autre reproduction ou représentation, en tout ou partie, sous quelque forme et de quelque manière que ce soit, est interdite sauf accord préalable et écrit de l'éditeur, en dehors des cas prévus par la législation en vigueur en France. II est précisé que son stockage dans une base de données est également interdit. 


\title{
Propriété industrielle et santé publique
}

\author{
Maurice Cassier
}

Comment redéfinir l'extension et les limites du droit de propriété et des brevets au regard du droit à la santé des populations? Le procès de Pretoria et l'exemple brésilien, à propos de l'épidémie de sida et des médicaments génériques, permettent de mieux cerner les termes du débat.

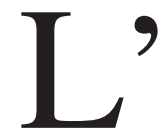

9 instauration du droit des brevets en France remonte à 1791. Elle fut justifiée par la reconnaissance de la propriété de l'inventeur sur son idée : propriété considérée comme la plus sacrée par les Constituants. Elle répondait aussi à la demande pressante des nouvelles compagnies industrielles de la fin du XVIII ${ }^{e}$ siècle de protéger leurs innovations et leurs marchés. Mais si la loi sur les brevets de 1791 ne comportait aucune exemption - toutes les catégories d'inventions pouvaient être juridiquement appropriées -, sa révision en 1844 déboucha sur l'exclusion des « compositions pharmaceutiques et remèdes de toutes espèces » de la sphère de la brevetabilité, et cela pour plus d'un siècle. Les arguments mobilisés par les partisans des brevets sur les médicaments, à l'instar de Gay-Lussac, et par les opposants, valent d'être rappelés. Gay-Lussac voulait favoriser l'émergence d'une nouvelle catégorie

\footnotetext{
Maurice Cassier est sociologue au Centre de Recherche Médecine, Sciences, Santé et Société (Cermes), unité mixte du Cnrs, de l'Inserm et de l'Ehess. Ses travaux portent sur l'intégration de la science et du marché dans le domaine de la recherche biomédicale et sur les relations entre propriété intellectuelle, innovation et santé publique, aujourd'hui et au XIXe siècle.
} 
d'inventeurs et encourager la transformation de l'industrie pharmaceutique, au-delà de la pharmacie d'officine qui prédominait jusqu'alors. Les opposants firent valoir les risques de l'établissement d'un monopole pour la production et la fourniture d'un bien jugé essentiel pour la population. Dans une logique de santé publique, on ne peut subordonner l'intérêt de la population à la propriété exclusive d'un inventeur ou d'un industriel. Le médicament n'est pas une marchandise comme les autres et la santé peut justifier une exclusion ou une suspension des droits de propriété industrielle. Les médicaments ont réintégré en France le droit commun des brevets en 1968 - après une phase de Brevet spécial du médicament entre 1959 et 1968. Il existe toujours, cependant, une mesure de suspension de ce droit de propriété, une licence d'office pour raison de santé publique, que le gouvernement français entend élargir aux inventions génétiques, notamment aux tests de diagnostic, dans un projet de loi présenté en octobre 2001.

On observe dans le monde une tendance à l'extension du droit des brevets sur les médicaments, accélérée par la conclusion en 1994 des accords de l'Omc sur la propriété intellectuelle ${ }^{1}$ : des pays qui ne reconnaissaient pas les brevets de médicaments (comme le Brésil) ou qui autorisaient seulement les brevets de procédés pharmaceutiques et non ceux sur les produits (comme l'Inde) ont été contraints d'adopter ou se préparent à adopter des brevets sur les molécules pharmaceutiques. Mais on enregistre parallèlement une confrontation accrue entre le droit des brevets et les demandes de santé des populations. L'année 2001 a été riche en débats : procès de Pretoria, plainte déposée à l'Omc par les Etats-Unis à l'encontre du Brésil, réunion de l'Omc à Doha. L'enjeu principal en était la fixation de l'étendue des droits de propriété dans le domaine de la santé, qui justifie une régulation particulière. Si les accords Adpic renforcent en effet le droit des brevets sur les médicaments, ils comportent aussi des dispositions d'exemption « pour protéger la santé et la vie des personnes » (article 27), ou de suspension de ces droits, «utilisations sans autorisation du détenteur du droit », dans des situations d'urgence nationale ou d'autres circonstances d'extrême gravité, ou en cas d'utilisation publique à des fins non commerciales (article 31). Ils prévoient que « les membres pourront [...] adopter les mesures nécessaires pour protéger la santé publique et la nutrition » (article 8). Entre les firmes pharmaceutiques et l'Afrique du Sud comme entre les Etats-Unis et le Brésil, l'enjeu était l'interprétation et la délimita- 
tion de ces clauses d'exemption pour raison de santé publique. Les mouvements constitués afin de combattre l'épidémie de sida jouent un rôle essentiel pour redéfinir l'extension et les limitations des droits de propriété au regard de la santé des populations. Certains Etats, à l'instar du Brésil, ont entrepris de créer un secteur hors marché pour produire et distribuer les molécules anti-sida. Des associations et des organisations humanitaires ont lancé des campagnes pour défendre l'accès des populations aux médicaments. Elles ont entrepris d'organiser des réseaux de fourniture de molécules génériques qui échappent aux marchés des médicaments brevetés. L'industrie pharmaceutique, quant à elle, défend ses brevets face à l'émergence « d'une idéologie des biens de l'humanité ${ }^{2}$ ». La Fédération pharmaceutique mondiale met en garde les gouvernements contre l'utilisation abusive des mesures de suspension des droits de propriété et contre l'émergence de circuits ou de secteurs pharmaceutiques en dehors du marché. Pour le directeur de l'Oms, « les droits de propriété industrielle stimulent la mise au point de nouveaux médicaments, mais doivent être appliqués de façon impartiale afin de protéger la santé publique ». L'analyse du procès de Pretoria et de l'expérience brésilienne en matière de médicaments génériques nous permettra de mieux cerner les termes du débat ainsi que les différents types de solutions, mises en œuvre ou proposées pour parvenir à de meilleurs équilibres entre propriété privée et bien commun en matière de santé.

\section{Pretoria : un Etat peut-il limiter la propriété privée sur le marché du médicament?}

En 1997, le gouvernement d'Afrique du Sud faisait voter le « Medicines and related Substances and Control Act ». Cette loi visait à mieux réguler le domaine du médicament - fabrication, évaluation, enregistrement, formation des prix, distribution, etc. L'un de ses articles (15 c) prévoit que le ministère de la Santé peut décider « dans certaines circonstances, de prendre des dispositions pour assurer l'offre de médicaments plus accessibles afin de protéger la santé du public ». Il peut notamment décider de limiter les droits des brevets relatifs à des médicaments protégés et commercialisés en Afrique du Sud. Il peut organiser des importations parallèles, afin de se procurer des médicaments vendus moins chers dans un autre pays par le détenteur des brevets ou des licences couvrant ces molécules. Ces dispositions furent aussitôt attaquées en justice par une coalition

2. Gilles Brisson, Pdg d'Aventis, lors d'un colloque à l'Institut de France sur

« Biosciences, risques, éthique et société » en octobre 2001. 
regroupant l'Association des fabricants pharmaceutiques d'Afrique du Sud et 39 firmes internationales. Celles-ci contestaient que le ministre puisse suspendre des brevets accordés en Afrique du Sud « compte tenu de certaines circonstances qui n'ont pas été définies par des règles ». Aux yeux des plaignants, cette loi autorisait le ministre de la Santé « à déposséder le détenteur d'une propriété intellectuelle relative à un produit pharmaceutique ou encore à l'exproprier d'une telle propriété sans compensation ». La motion mettait également en cause la procédure des importations parallèles, en introduisant « des discriminations en faveur des médicaments importés au détriment des fabricants locaux ». L'Afrique du Sud était accusée d'outrepasser les dispositions prévues dans les accords de l'Omc par « le recours automatique au système de la licence obligatoire »... On peut lire ce procès comme une mise à l'épreuve des accords Trips (Adpic) par l'industrie pharmaceutique qui tentait d'imposer une définition restrictive des « circonstances » susceptibles de justifier une suspension des droits de propriété sur ses marchés. Car l'Afrique du Sud représente un enjeu important, dans la mesure où les droits de propriété existants sur les médicaments y sont bien établis et où l'importance du marché justifie le dépôt de brevets de la part des firmes internationales qui, souvent, n'étendent pas leur propriété dans les autres pays de l'Afrique subsaharienne. Mais pour le gouvernement et plus encore pour les associations anti-sida, cette loi ouvrait des perspectives pour commencer à traiter l'épidémie de sida qui affecte 4,2 millions de personnes - 1 adulte sur 5 - et qui est susceptible, d'ici à 2015, de ramener l'espérance de vie dans ce pays à son niveau du début du $\mathrm{XX}^{\mathrm{e}}$ siècle.

En avril 2001, devant l'extension des protestations qui affirmèrent les « droits des malades » face au « droit des brevets $»^{3}$, le retrait de la plainte des laboratoires pharmaceutiques marqua une certaine reconnaissance internationale du bien commun en matière de santé. En dépit des offensives menées durant l'été 2001 par l'industrie pharmaceutique pour tenter de réduire les dispositions d'exception, la réunion interministérielle de Doha déboucha sur une nouvelle affirmation de ces mesures. La Déclaration de Doha ${ }^{4}$ enregistre tout d'abord le rôle ambivalent du système du brevet, instrument d'incitation à développer de nouveaux médicaments, et dont « les effets sur les prix » peuvent restreindre leur accessibilité. Elle réaffirme « les flexibilités » contenues dans l'accord Adpic, notamment le droit de chaque membre « d'accorder des licences obligatoires et la liberté de déterminer les motifs pour lesquels de telles licences sont accordées ». C'est le contre-pied de la position défendue par les firmes pharmaceutiques lors du

3. Comme l'affichait un dessin politique paru en Afrique du Sud pendant le procès. 4. L'accord sur la propriété intellectuelle et la santé publique, publié en novembre 2001 . 
procès de Pretoria. La portée juridique de cette déclaration est encore à déterminer, mais d'autres Etats comme le Kenya ont adopté des lois sur la propriété industrielle comportant des mesures d'exception pour raison de santé publique.

\section{Initiatives d'associations}

En Afrique du Sud, le gouvernement a rédigé des règles pour mettre en œuvre la loi de 1997, dont l'application était suspendue par le procès. Les associations anti-sida demandent l'utilisation immédiate de la procédure des licences obligatoires afin de commencer à produire localement ou à importer des molécules génériques. Le gouvernement, cependant, en est resté à une position très prudente. Il propose de mettre en place des importations parallèles, pour bénéficier des différences de prix des molécules commercialisées «par ou avec l'accord du détenteur du brevet », ce qui exclut pour le moment des licences non-volontaires. Il n'a pas donné suite à la proposition du laboratoire indien Cipla qui demandait l'attribution de licences obligatoires pour 8 molécules anti-sida, qui auraient été importées en Afrique du sud. De même, il a décliné une offre thailandaise de transférer une technologie libre de droits pour produire 300 médicaments génériques. L'industrie pharmaceutique s'est réjouie de la prudence du gouvernement sud-africain, assurée que celui-ci « respectera les droits sur les brevets ${ }^{5}$ ». Deux firmes ont proposé des licences volontaires à un laboratoire sud-africain, Aspen, pour produire des antirétroviraux. Toutefois, les associations de malades critiquent les termes trop restrictifs de ces licences exclusives. Les prix demeureront supérieurs aux prix de référence des laboratoires de génériques brésiliens.

Ces débats ont vu apparaître les associations de malades ou les organisations humanitaires sur le terrain de la propriété industrielle. Treatment Access Campaign (Tac) a été fondée en 1998 avec l'objectif d'intervenir pour élargir l'accessibilité des traitements pour les malades du sida. L'association rappelle que «l'intérêt de la santé doit toujours prévaloir sur les intérêts du commerce ${ }^{6} \gg$. Reconnue partie civile dans le procès de Pretoria, elle a soutenu le Medicines Act. Elle milite pour le recours à des importations de médicaments génériques en provenance de Thaillande, d'Inde ou du Brésil, en se fondant sur des tableaux comparatifs des prix des molécules produites sous brevet et hors brevet. Tac a organisé des importations de molécules

5. Pharmaceutical Manufacturers Association, avril 2001.

6. Document de TAC sur les brevets pharmaceutiques, les droits de l'homme et l'épidémie de sida. 
génériques fabriquées en Thaillande et au Brésil, en enfreignant les brevets sud-africains qui protègent ces médicaments. Elle demande que le gouvernement sollicite les propriétaires des molécules pour attribuer des licences volontaires et, en cas de refus, décide de licences obligatoires. Tac a enfin engagé des poursuites contre le ministre de la Santé sud-africain devant la réticence du gouvernement à organiser la distribution d'un médicament, la névirapine, pour diminuer le taux de transmission du VIH entre la mère et l'enfant. Quant à Médecins sans frontières (Msf), elle a pris deux initiatives qui ont connu un grand retentissement. Tout d'abord, elle a négocié, en février 2001, avec le laboratoire Cipla un accord d'approvisionnement pour une tri thérapie annuelle à un prix 30 fois inférieur à celui du marché des molécules équivalentes sous brevet (350 dollars contre 10 à 15000). Ensuite, Msf Afrique du Sud est intervenue auprès de l'Université de Yale, détentrice du brevet d'un des antirétroviraux pour lui demander d'accorder à l'Afrique du Sud une licence volontaire. Cette intervention, qui a provoqué un vif débat à l'Université de Yale, interrogeait la politique de propriété industrielle des institutions académiques, notamment l'attribution de licences exclusives sur leurs inventions médicales. Enfin, Msf finance l'importation de tri thérapies en provenance du Brésil qu'elle distribuera dans le cadre d'une opération pilote.

\section{La production brésilienne de médicaments génériques}

Le Brésil a trouvé une articulation originale entre logique industrielle et logique de santé publique. Des laboratoires publics produisent des molécules hors brevet qui sont achetées et distribuées gratuitement par le ministère de la Santé à 100000 malades aujourd'hui (sur 196000 cas de sida recensés). Cette expérience a bénéficié au départ d'une situation particulière : avant 1997, les médicaments n'étaient pas brevetables au Brésil et il était donc licite de copier les molécules protégées. Les laboratoires publics de plusieurs Etats brésiliens ont profité de cette fenêtre en termes de propriété pour s'engager dans la copie et la production locale de plusieurs antirétroviraux, à partir de 1994. Des chimistes transfuges des laboratoires pharmaceutiques ont utilisé leur savoir-faire pour organiser le processus de copie. Les molécules produites sont soumises à des essais de bio-équivalence pour l'attribution du statut officiel de médicament générique. Les laboratoires publics brésiliens sont aujourd'hui en mesure de fabriquer une douzaine d'antirétroviraux et de les fournir à des prix très en-dessous du marché. Cette offre a entraîné une chute moyenne des prix de $82 \%{ }^{7}$. 
Lorsqu'ils répondent à des appels d'offre du ministère de la Santé brésilien, les laboratoires internationaux sont contraints de s'aligner sur les prix brésiliens, qui servent de référence. Ainsi, Bristol Myers a proposé une de ses molécules trois fois moins cher qu'aux Etats-Unis et même moins cher que la copie brésilienne.

Cette politique de production locale s'inscrit dans une politique de santé publique. C'est ce qui différencie l'expérience brésilienne de l'expérience indienne où des laboratoires privés produisent des molécules hors brevet, à bas prix, mais dans le strict contexte du marché des médicaments. En 1996, le ministère de la Santé du Brésil a édicté un décret sur l'accès universel aux molécules anti-sida, suivi par une loi sur les médicaments génériques adoptée en 1998. En 2001, le ministère de la Santé a dépensé 422 millions de dollars pour acheter et distribuer les molécules anti-sida. Ce programme qui couple accès universel aux médicaments, approvisionnement centralisé, production publique, a déjà eu des effets importants en termes de santé publique : réduction de moitié de la mortalité, réduction de la morbidité, réduction des coûts d'hospitalisation (234000 hospitalisations économisées entre 1997 et 2000, soit une économie de 677 millions de dollars, selon le ministère de la Santé).

Naturellement, la mise en place de ces circuits de production hors brevet et hors marché a provoqué de vives réactions de l'industrie pharmaceutique et des Etats-Unis. Les laboratoires internationaux ont exigé l'adoption d'une législation qui reconnaisse les brevets de médicaments, sous peine de geler leurs investissements. L'adoption des accords Adpic par le Brésil allait dans le même sens. Une nouvelle loi fut votée en 1997. Mais elle ne compromet pas entièrement la production de copies. Premièrement, elle ne protège que les nouveaux médicaments : les laboratoires brésiliens peuvent continuer à copier librement la première génération d'antirétroviraux. Deuxièmement, pour les nouvelles molécules qui tombent sur le coup des brevets, la nouvelle loi exige qu'elles soient fabriquées au Brésil dans un délai de trois ans après leur introduction sur le marché. Si le détenteur de la molécule ne met pas en place de production locale, il sera tenu de fournir sa formule et d'en permettre la production. En compensation, il percevra des royalties. Cette clause fut attaquée devant l'Omc par les Etats-Unis en février 2001, au motif qu'elle introduit une discrimination entre les produits brevetés et les molécules produites localement. Mais après la retraite des firmes pharmaceutiques du procès de Pretoria, les Etats-Unis ont décidé de retirer leur plainte. Le ministère de la Santé brésilien n'hésite pas à menacer de recourir à une licence obligatoire lorsqu'il négocie les prix des molécules avec les grands laboratoires. La menace est d'autant plus crédible qu'il dispose d'un potentiel technologique prêt à prendre en charge la production. 


\section{Quels compromis entre propriété privée et bien commun?}

Face à la demande de plus en plus forte d'une accessibilité universelle des médicaments et à la demande des plusieurs Etats du Sud de pouvoir utiliser des mesures de suspension des brevets pour protéger leurs populations, les firmes pharmaceutiques ont avancé des contre-propositions : dons, réductions de prix (dans certains cas jusqu'à $80 \%$ des prix pratiqués en Europe ou en Amérique du Nord), voire une politique systématique de prix différentiels entre pays du Nord et du Sud. Pour les firmes, ces mesures présentent l'avantage de ne pas empiéter sur leurs droits de propriété et leur pouvoir de marché, et de repousser les mesures de contrôle des prix, prévues dans la loi sud-africaine, ou de licences obligatoires. Cependant, les populations et les Etats demeurent entièrement dépendants de l'étendue de ces concessions. Les associations de malades sud-africaines critiquent l'insuffisance des dons ou des baisses de prix proposées ou encore les termes des licences offertes aux laboratoires locaux. Les grands laboratoires craignent eux-mêmes que les prix différentiels appliqués au sud deviennent des prix de référence pour les pays du nord.

Des économistes ont imaginé plusieurs dispositifs : le rachat des droits de propriété sur certains médicaments par une institution mondiale - par exemple l'OMS, avec la difficulté de fixer et de financer la rente d'innovation -, ou bien un système qui conduirait les firmes pharmaceutiques à opter pour une protection dans un pays du nord à l'exclusion d'une protection dans un pays du sud. Dans ce dernier cas, les laboratoires indiens, par exemple, pourraient librement copier la molécule. Cette politique offrirait un bénéfice immédiat aux populations du sud sans détruire les incitations au développement des molécules. Pour J. Lanjouwe, « cette politique serait entièrement contrôlée par le gouvernement américain. A l'opposé du système des licences obligatoires décidées par des gouvernements sous la pression d'intérêts locaux ».

Le modèle brésilien dans le domaine du sida met au contraire en avant le pouvoir et l'intervention des Etats, notamment du ministère de la Santé. Il repose sur une absence de droits de propriété sur les médicaments - avant 1997 - ou sur des mesures de limitation de ces droits - pour produire localement les molécules mises sur le marché -, sur la production publique de certaines molécules, sur la mise en œuvre d'une politique de santé publique prévoyant l'achat et la distribution gratuite des médicaments aux malades. C'est-à-dire la mise en place de circuits hors marché justifiés par la gravité d'une épidémie. Ces pouvoirs d'intervention de l'Etat licences obligatoires - et l'existence d'un secteur public de production de médicaments génériques jouent un rôle régulateur pour le marché brésilien et mondial. Les courbes sont éloquentes : au Brésil, les prix des molécules 
se mettent à baisser dès lors que la production locale de molécules génériques commence; en revanche, les prix des molécules ne font que légèrement baisser ou restent stables dès lors que l'offre des grands laboratoires n'est pas confrontée à celle des laboratoires publics brésiliens. Les laboratoires privés indiens qui produisent hors brevets jouent un rôle similaire.

L'institution de la santé comme un bien commun suppose de définir de nouveaux équilibres entre la propriété privée et l'intérêt des populations. Le bien commun n'est pas spontanément satisfait par le seul jeu de la propriété et du marché. Les incitations privées, à l'instar du brevet, sont susceptibles de produire de fortes asymétries au bénéfice du propriétaire exclusif de la molécule et le cas échéant des situations de blocage dans l'accès aux médicaments. Ce constat justifie l'intervention des Etats pour réintroduire de la « sagesse civique » (Keynes) dans la production et la fourniture de médicaments, par le jeu de procédures d'exemption ou de suspension des droits de brevets, de dispositifs de contrôle de la formation des prix, ou par la création de circuits en dehors du marché. Les pays du sud doivent se saisir de la flexibilité des accords Adpic sur la propriété intellectuelle, ce qu'ils ne font pas toujours ${ }^{8}$. Mais la notion de bien commun est beaucoup plus large que celle de l'intérêt de l'Etat ou des organisations inter étatiques. L'intervention des associations de malades, des organisations humanitaires, des syndicats de salariés, des professionnels de santé sur les problèmes de propriété industrielle et d'accès aux médicaments joue un rôle essentiel pour imposer et construire concrètement ces biens communs.

Maurice Cassier

8. Cf. les récents accords de Bangui adoptés par les Etats d'Afrique de l'Ouest et du Centre. 